\title{
Clostridium perfringens Sialidases: Potential Contributors to Intestinal Pathogenesis and Therapeutic Targets
}

\author{
Jihong Li ${ }^{1}$, Francisco A. Uzal ${ }^{2}$ and Bruce A. McClane ${ }^{1, *}$ \\ 1 Department of Microbiology and Molecular Genetics, University of Pittsburgh School of Medicine, \\ Room 420, Bridgeside Point II Building, 450 Technology Drive, Pittsburgh, PA 15219, USA; jihongli@pitt.edu \\ 2 California Animal Health and Food Safety Laboratory, San Bernardino Branch, School of Veterinary \\ Medicine, University of California-Davis, San Bernardino, CA 92408, USA; fauzal@ucdavis.edu \\ * Correspondence: bamcc@pitt.edu; Tel.: +1-412-648-9022
}

Academic Editor: Holger Barth

Received: 28 October 2016; Accepted: 13 November 2016; Published: 19 November 2016

\begin{abstract}
Clostridium perfringens is a major cause of histotoxic and intestinal infections of humans and other animals. This Gram-positive anaerobic bacterium can produce up to three sialidases named NanH, NanI, and NanJ. The role of sialidases in histotoxic infections, such as gas gangrene (clostridial myonecrosis), remains equivocal. However, recent in vitro studies suggest that NanI may contribute to intestinal virulence by upregulating production of some toxins associated with intestinal infection, increasing the binding and activity of some of those toxins, and enhancing adherence of C. perfringens to intestinal cells. Possible contributions of NanI to intestinal colonization are further supported by observations that the $C$. perfringens strains causing acute food poisoning in humans often lack the nanI gene, while other $C$. perfringens strains causing chronic intestinal infections in humans usually carry a nanI gene. Certain sialidase inhibitors have been shown to block NanI activity and reduce $C$. perfringens adherence to cultured enterocyte-like cells, opening the possibility that sialidase inhibitors could be useful therapeutics against $C$. perfringens intestinal infections. These initial in vitro observations should be tested for their in vivo significance using animal models of intestinal infections.
\end{abstract}

Keywords: Clostridium perfringens; intestinal infections; gas gangrene; toxins; sialidases; sialidase inhibitors

\section{An Introduction to Clostridium perfringens}

Clostridium perfringens is present throughout the environment, including soil, sewage, feces, foods, and the normal gastrointestinal flora of animals [1,2]. This Gram-positive, anaerobic, spore-forming bacterium is also a feared pathogen of both humans and other animals [2,3]. The most notable C. perfringens histotoxic infection is the rapidly-fatal human disease named clostridial myonecrosis (traumatic gas gangrene) [4,5]. This bacterium is also a preeminent cause of common, and sometimes lethal, infections originating in the intestines of humans or livestock $[2,6]$. Those intestinal infections often involve damage to the small intestine, or to both the small intestine and colon, which results in enteritis or enterocolitis, respectively $[1,2]$. C. perfringens intestinal infections can also progress to enterotoxemia, where a toxin(s) is produced in the intestines and then absorbed to affect extraintestinal organs such as the brain $[2,7]$.

The virulence of this bacterium involves its ability to produce a vast toxin armory $[2,3,8]$. Currently $\sim 20$ different $C$. perfringens toxins have been identified, with more likely awaiting discovery [8-15]. Toxin production repertoires vary greatly among different $C$. perfringens strains, 
permitting classification of these isolates into five types (A-E), based upon an isolate's production of four typing toxins (alpha, beta, iota, and epsilon toxins) (Table 1) [9,10].

Table 1. C. perfringens typing table.

\begin{tabular}{|c|c|c|c|c|}
\hline \multirow{2}{*}{ Type } & \multicolumn{4}{|c|}{ Toxin Production $^{a}$} \\
\hline & $\alpha$ & $\beta$ & $\varepsilon$ & l \\
\hline A & + & - & - & - \\
\hline B & + & + & + & - \\
\hline $\mathrm{C}$ & + & + & - & - \\
\hline $\mathrm{D}$ & + & - & + & - \\
\hline $\mathrm{E}$ & + & - & - & + \\
\hline
\end{tabular}

C. perfringens type designations correlate with disease causation, as shown in Table 2. Two typing toxins, i.e., beta toxin (CPB) and epsilon toxin (ETX), have proven importance in C. perfringens intestinal infections of mammalian livestock $[3,16,17]$. C. perfringens produces other toxins that, while not used for typing classification, are nonetheless important for infections originating in the intestines of agriculturally-important animals. The foremost example is necrotic enteritis B (NetB) toxin, which is critical when $C$. perfringens causes avian necrotic enteritis in poultry [12].

Table 2. Diseases associated with the major types/subtypes of $C$. perfringens.

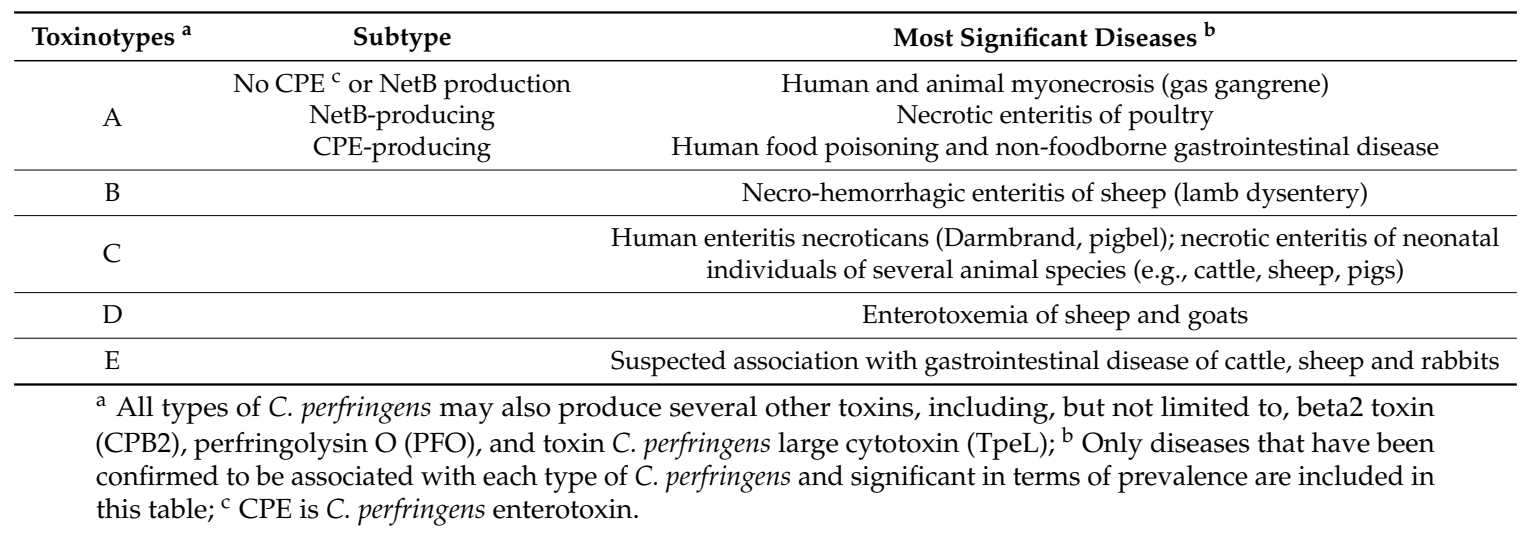

With respect to human C. perfringens infections, type A strains are responsible for causing most histotoxic infections. During gas gangrene, alpha toxin (CPA) plays the major role in virulence. A non-typing toxin named PFO also contributes to this disease $[4,5]$.

To date, only type $\mathrm{A}$ and $\mathrm{C}$ strains of $\mathrm{C}$. perfringens have been conclusively linked to human diseases originating in the intestines [1-3,18]. Type $C$ strains use their CPB to cause enteritis necroticans (EN), which was first described in post-World War II Germany, where it was referred to as darmbrand [18-20]. In the 1960s-1970s, EN, known locally as pigbel, was a major cause of death of children in the Papua New Guinea (PNG) Highlands [20,21]. Pigbel develops in children with reduced trypsin levels due to predisposing conditions, including malnutrition, a diet rich in sweet potato (which contains a trypsin inhibitor), and/or intestinal infections with pathogens producing a trypsin inhibitor [20,21]. Their low intestinal trypsin levels render these children susceptible to infection by type $C$ strains because normal trypsin levels would otherwise easily inactivate CPB when it is produced in the intestines. Consequently, children suffering from pigbel develop CPB-induced necrotic enteritis or enterotoxemia and often die rapidly. The only treatment for pigbel is resection of the bowel; however, this surgical intervention is only effective if performed early after the onset of 
infection [20,21]. A vaccine introduced in the 1980s dropped the incidence of pigbel dramatically in PNG. Unfortunately, pigbel vaccination has since decreased and this illness may now be reappearing.

Although not used for typing classification, CPE is the toxin responsible for causing the gastrointestinal symptoms of $C$. perfringens type A food poisoning (FP) [1,22]. This FP is currently the 2nd most common bacterial foodborne disease in the USA, where one million cases occur annually and economic losses approach $\$ 500$ million/year [1,23]. In people with fecal impaction or severe constipation due to side-effects from medications used to treat other pre-existing conditions, C. perfringens type A FP can be much more severe and is often fatal [1,24]. Studies with animal models suggest this may be due to absorption of CPE from the intestines, resulting in an enterotoxemia that involves the liver and kidneys [24]. CPE-producing type A strains also cause about $5 \%-15 \%$ of all cases of nonfoodborne human GI diseases, most notably antibiotic-associated diarrhea (AAD) [1,25].

As discussed later, $\mathrm{CPE}$-associated AAD cases are more severe and longer lasting than typical cases of $C$. perfringens type A FP, which usually self-resolve within $24 \mathrm{~h}[1,25]$. While the cpe gene can be either chromosomal or plasmid-borne in type A strains, most ( $70 \%)$ type A FP strains carry a chromosomal cpe gene [1]. In contrast, nearly $100 \%$ of type A AAD strains carry a plasmid cpe gene [1]. There are also many other genetic differences between these two groups of type A cpe-positive groups. For example, type A strains carrying a chromosomal cpe gene produce a unique small acid soluble protein (SASP4) variant that provides their spores with greater resistance to heat, cold, and chemical treatment and probably facilitates survival of these bacteria in the food environment $[1,26,27]$. Other differences between type A chromosomal cpe strains and type A plasmid cpe strains, particularly with regard to sialidase gene carriage, will be discussed later.

In addition to its essential role during intestinal infections by type A cpe-positive strains, CPE may also contribute to some cases of human EN caused by type C strains [18]. However, CPB is clearly of critical importance for the pathogenesis of this disease [16].

\section{C. perfringens Sialidases}

Sialic acids are a carbohydrate family containing about 40 different nine-carbon relatives of neuraminic acid [28,29]. Under physiological conditions, sialic acids are negatively charged [29]. $\mathrm{N}$-acetylneuraminic acid (Neu5Ac), whose amino group is acetylated, is the most widespread sialic acid $[29,30]$. Sialic acids are important components of the serum and mucus and represent the terminal sugar residue of many glycan chains on host cell surfaces, where they are involved in cell- cell recognition. Sialic acids can also stabilize enzymes or cell membrane proteins. Finally, due to their negative charge, sialic acids can mediate binding and transport of positively-charged molecules [28-30].

Sialidases, also referred to as neuraminidases (E.C.3.2.1.18), are key enzymes that hydrolyze the $\alpha$-linkage of terminal sialic acids on various sialoglycoconjugates to generate free sialic acid [28-31]. Sialidases are made by certain viruses, microorganisms, and vertebrate animals, but not by plants. Included amongst the sialidase-producing viruses and bacteria are several prominent pathogens, e.g., influenza virus, Vibrio cholerae, Streptococcus pneumoniae, and C. perfringens. Sialidases can have a nutritional function for normal flora or pathogenic bacteria [28,29,31]. In addition, they often function directly as virulence factors during bacterial pathogenesis [30,32], as will be discussed later for C. perfringens.

C. perfringens produces three different sialidases, which are named NanH, NanI, and NanJ [33]. $\mathrm{NanH}(43 \mathrm{kDa})$ lacks a secretion signal peptide and thus has a cytoplasmic location in log-phase cultures [33,34]. In contrast, NanI (77 kDa) and NanJ (129 kDa) are secreted exosialidases ([33], Figure 1). The catalytic modules of all three sialidases show conserved amino acid sequence identity and belong to the family 33 carbohydrate binding module (CBMs, Figure 1) [35]. Compared to $\mathrm{NanH}$, which only consists of a catalytic domain, NanI and NanJ also possess additional accessory carbohydrate-binding modules [35]. It is thought that these carbohydrate-binding domains increase the binding affinity between NanI and NanJ and their polyvalent substrates. NanJ has a complex multimodular structure comprised of a central catalytic module and five accessory modules [35]. 
The two N-terminal modules show amino acid sequence identity with family 32 and family 40 CBMs. NanI has a simpler structure consisting of a catalytic module and an N-terminal family 40 CBM [35].

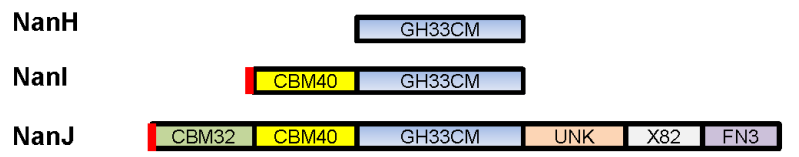

Figure 1. Modular organization of the C. perfringens sialidases. CBM32 is a carbohydrate binding module (CBM) belonging to the family 32 CBM; CBM40 is a module having an amino acid sequence identity with a family $40 \mathrm{CBM}$; GH33CM is a family $33 \mathrm{CBM}$; UNK is a module having unknown function; X82 is a family 82 "X module" of unknown function; FN3 is a module sharing distant identity with fibronectin type III domains. The red small boxes are secretion signals. Modified with permission from [35]. Copyright 2007 American Chemical Society.

Most C. perfringens strains produce all three sialidases (further discussion below). However, as discussed in more detail later, some C. perfringens strains produce only one or two of the three sialidases. For strains producing all three sialidases, NanI is usually responsible for $\sim 70 \%$ of total exosialidase activity [33,34,36,37].

To characterize the properties of the three C. perfringens sialidases, a recent study constructed a series of isogenic mutants, where two of the three sialidase genes present in C. perfringens type D strain CN3718 were inactivated [33]. This strategy created mutants that were each expressing, at their native levels, only NanJ, NanI, or NanH in a background free of contamination from the other two sialidases to allow a precise characterization of the enzymatic properties of each sialidase. NanI was found to be more heat-tolerant compared to $\mathrm{NanJ}$ or $\mathrm{NanH}$, both of which exhibited greatly reduced sialidase activity at temperatures above $43{ }^{\circ} \mathrm{C}$ [33]. In this experimental system, all three sialidases worked best at low $\mathrm{pH}$ conditions ( $\mathrm{pH} \sim 5)$. The enzyme activity of each sialidase was shown to vary in sensitivity to various metal ions [33]. Furthermore, unlike the sialidases from Streptomyces spp., C. perfringens sialidases were found to be sensitive to $\mathrm{p}$-chloromercuribenzoate, which reacts with thiol groups in proteins [33]. Finally, the three C. perfringens sialidases showed different substrate preferences. NanI exhibited preferential activity in the order of $\alpha-2,3>\alpha-2,6>\alpha-2,8$ sialic acid linkages and was responsible for most of the activity in CN3718 supernatants that was directed against those sialic acid linkages. NanJ showed a preference for $\alpha-2,6>\alpha-2,8>\alpha-2,3$ sialic acid linkages. Finally, NanH activity was strongest for $\alpha-2,8>\alpha-2,3>\alpha-2,6$ sialic acid linkages [33]. This diversity in linkage preferences suggests that, when present together, the three $C$. perfringens sialidases work in combination to generate free sialic acid, even from complex substrates [33].

\section{C. perfringens Sialidases: Genetics and Regulation of Expression}

All three C. perfringens sialidases are encoded by chromosomal genes, although those genes are located in different regions of the chromosome [38,39]. The sialidase-encoding ORFs in many C. perfringens strains have now been sequenced. Those sequencing analyses indicated that, amongst different $C$. perfringens strains, the NanJ sequence shares $96 \%$ to $100 \%$ identity, the NanI sequence has $98 \%$ to $100 \%$ identity, and the NanH sequence shares $93 \%$ to $100 \%$ identity $[34,38,39]$.

As true for their toxin production, C. perfringens strains vary in their patterns of sialidase production. Most strains produce all three sialidases, with NanI usually being responsible for most of the sialidase activity in culture supernatants of those C. perfringens strains [34,36]. However, NanI production is not essential for $C$. perfringens growth since some strains of this bacterium naturally lack the nanI gene [36]. Interestingly, the nanI gene is consistently absent from the type A FP strains carrying a chromosomal cpe gene, as well as the genetically related type C darmbrand strains [36]. In contrast, the nanI gene is carried by most plasmid cpe-carrying type A AAD strains, cpe-negative type A normal human intestinal flora strains, and type C pig-bel strains [36]. Since NanI is usually the 
major sialidase of $C$. perfringens, it is not surprising that exosialidase activity is typically significantly lower for naturally nanI-negative strains compared to strains carrying a nanI gene [36]. The potential pathogenic importance of these differences will be discussed later.

Most type A FP strains with a chromosomal cpe gene lack the nanJ gene, as well as the nanI gene. However, those typical FP strains do carry the nanH gene. It should be noted that occasional strains of C. perfringens besides the type A chromosomal cpe FP strains or type C darmbrand strains also lack a sialidase gene. For example, Strain 13 is a cpe-negative type A strain that can cause gas gangrene yet it lacks the nanH gene $[37,40]$.

The regulation of sialidase production by $C$. perfringens is complicated (Figure 2). A number of regulators influencing expression of one or more sialidase genes have been identified. For example, the VirS/VirR two-component signal transduction system was shown to upregulate nanI and nanj expression [41]. Rather than a direct regulatory effect involving VirS binding to sialidase gene promoters, this VirS/R two-component system positively controls expression of the vrr gene, which encodes virR-regulated RNA (VR-RNA). This regulatory RNA then modulates sialidase gene expression [42]. ReeS is another sensor kinase whose presence also increases nanI and nanJ sialidase gene expression by a putative response regulator, referred to as ReeR [43]. In contrast, a transcriptional regulator named RevR has different regulatory effects on nanJ and nanI expression, possibly via a proposed sensor kinase named RevS. RevR increases nanJ expression, but negatively regulates nanI expression [44]. Finally, using a codY null mutant of C. perfringens type D strain CN3718, our group showed that CodY represses NanJ and NanH production, although it does not affect NanI production [45].

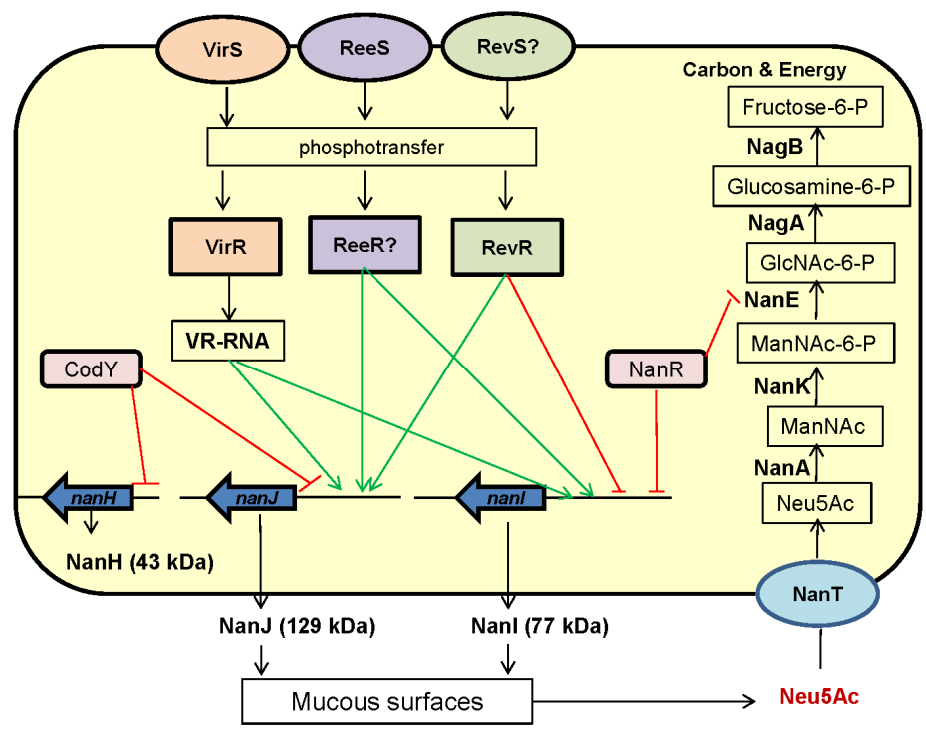

Figure 2. A proposed model for regulation of expression of sialidase genes and the pathway for sialic acid metabolism in C. perfringens. Exosialidases generate free sialic acid from mucus or host cell surfaces $[33,40]$. The free sialic acid is then transported into C. perfringens where it is metabolized to fructose-6-P [40]. There is evidence that VirS/VirR, RevR, ReeS, NanR, and CodY systems directly or indirectly affect sialidase production, although inter-relationships between these regulators are unclear [40,41,43-46]. The VirS/VirR two component system acts as a positive regulator of the vrr gene, which encodes VR-RNA. VR-RNA is then a positive regulator of nanI and nanJ expression [41,42]. The ReeS sensor kinase positively regulates nanI and nanJ gene expression, presumably by a putative transcriptional regulator named ReeR [43]. RevS positively regulates nanJ expression but negatively regulates nanI expression [44]. CodY represses nanH and nanJ expression [45]. Based on sequence homology comparisons with similar regulators in other bacteria, NanR may repress nanI expression and the sialic acid metabolism pathway, but there is no direct experimental evidence yet to support this hypothesis. Green lines indicate positive regulation while red lines indicate negative regulation. 
No proven regulator has yet been identified that directly modulates sialidase production by binding to the promoters of $C$. perfringens sialidase genes. However, gel mobility shift assays have demonstrated high affinity binding of a purified protein named NanR to DNA from the promoter region of the nanI gene [40]. Since NanR has homology with the ribose-5-phosphate isomerase B regulator (RpiR) family of transcriptional repressors known to control sialidase production in bacteria such as E. coli, Vibrio vulnifcus, and Staphylococcus aureus, this result suggests that NanR may be involved in nanI expression regulation $[31,40,47]$. Consistent with its potential involvement in sialic acid generation and usage, NanR lies within a six-gene operon encoding the complete pathway for transport and metabolism of sialic acid by C. perfringens [40]. As discussed in more detail below, this operon also encodes NanE (epimerase) and NanA (sialic acid lyase) enzymes.

For the nanI and nanJ genes, primer extension analyses identified three or two putative transcription start sites, respectively [40]. These promoters are located within $\sim 500$ bp of the start codons of nanI and nanJ. This multiplicity of promoters may provide one explanation for why so many different regulators control sialidase expression, although (as mentioned) detailed understanding of regulator binding to these promoter sequences is currently lacking [40].

Only a limited number of bacterial species, mainly those having a close association with the sialic acid-rich environment of the host, can utilize Neu5Ac [48]. C. perfringens was actually the first bacterium demonstrated to be capable of utilizing a sialic acid (Neu5Ac) as a carbon source [31]. This result was later confirmed by another study showing that Neu5Ac can be used by C. perfringens when growing in a semi-defined medium [31]. It was also recently found that NanI and NanJ, but not NanH, can cause the release of sialic acid from Caco-2 cells [33]. This effect may contribute to pathogenesis, since these sialidases could help C. perfringens obtain nutrients in vivo by releasing sialic acid from glycolipids or glycoproteins on the host cell surface or in mucus. Interestingly, contact with Caco-2 cells was shown to upregulate the expression of NanI [34], which could potentiate NanI contributions during intestinal infections, as discussed later.

NanI, but not NanJ, production is induced by the addition of Neu5Ac to a medium [40]. As introduced earlier, NanR is part of an operon that encodes a complete pathway for the transport and metabolism of sialic acid. After sialic acid is generated by sialidases, it is then transported and metabolized by products of this operon, also referred to as the Nan cluster. This process involves an initial conversion of sialic acid to $\mathrm{N}$-acetyl glucosamine, followed by metabolism of that carbohydrate to fructose-6-P, which C. perfringens can use as carbon sources and for energy production (Figure 2) [40,46].

Many other pathogenic and commensal bacteria found in the intestines carry a Nan cluster for sialic acid utilization [48]. Examples of such bacteria include Vibrio cholerae and Salmonella enterica $[48,49]$. Many of these bacteria capable of utilizing sialic acid also colonize the human or animal intestinal tract. This correlation is probably not a coincidence since sialic acid is a component of mucin, the major protein in mucus, which is abundant in the human and animal intestines [48]. However, to generate free sialic acid from mucus for uptake and metabolism inside the bacterial cell, extracellular sialidases must be present in the intestinal environment. Interestingly, some pathogenic bacteria, e.g., C. difficile, have a Nan cluster but do not produce their own sialidase. Instead, it is believed that $C$. difficile uses free sialic acid generated in the intestines by sialidases produced by other bacteria, such as Bacteroides thetaiotaomicron [50,51].

\section{Possible Contributions of Sialidases to C. perfringens Diseases}

Some pathogens use sialic acids to coat their cell surface, the flagellum, the capsule polysaccharide, or the lipopolysaccharide. This masks these bacteria so they can avoid the host immune system defense [28]. Whether C. perfringens coats its surface with host-derived sialic acid has not been studied, to our knowledge.

Sialidases can also promote in vivo growth and colonization of bacterial pathogens [30]. For extraintestinal infections, an example is the major human respiratory tract pathogen Streptococcus pneumoniae, which encodes up to three sialidases, named NanA, NanB, and NanC. 
NanA is the predominant sialidase that removes the sialic acid Neu5Ac from a variety of glycoconjugates. NanB is an intramolecular trans-sialidase producing 2,7-anhydro-Neu5Ac selectively from $\alpha 2,3$-sialosides, while NanC produces 2-deoxy-2,3-didehydro- $N$-acetylneuraminic acid (Neu5Ac2en), which can be hydrated to Neu5Ac. The three pneumococcal sialidases share a common catalytic mechanism up to the final product formation step, and all three sialidases are implicated in pathogenesis, including colonization, and are potential drug targets [52]. S. pneumoniae then takes up and metabolizes sialic acid using a similar pathway as present in C. perfringens [40].

The contribution of sialidases to growth and colonization during extraintestinal infections caused by $C$. perfringens is unclear. One study demonstrated that a nanJ and nanI double null mutant of C. perfringens strain 13 remains fully virulent in the mouse myonecrosis model [37]. This result could suggest that Neu5Ac metabolism is not essential for growth or colonization by C. perfringens in muscle. However, as noted in that study [37], this result does not necessarily preclude subtle contributions of sialidases to colonization or growth since the mouse myonecrosis model requires a massive inoculum that may mask such contributions.

Increasing evidence indicates that sialidases and sialic acid often play significant roles in growth and colonization of the intestines by bacterial pathogens $[34,49,52,53]$. For example, sialic acid appears to be an important source of carbon and energy for survival, growth and adherence of $E$. coli in the gastrointestinal system [54]. Similarly, catabolism of sialic acid by $V$. cholerae plays a significant role in both in vitro and in vivo colonization and growth [49]. In addition, $V$. cholerae sialidase enhances the binding and uptake of cholera toxin [55]. In V. vulnificus, a Nan utilization system is also important for bacterial colonization and growth in the intestines [56]. The sialidase NanS play a role in Clostridium sordellii adhesion and enhances Non-TcsL mediated cytotoxicity [53]. As mentioned above, $C$. difficile uses sialic acid generated by sialidases of other bacteria to grow and persist in the intestines [50,51].

As mentioned earlier, NanI may contribute to in vivo growth. Contact of $C$. perfringens with Caco-2 cells increases the production of this enzyme [34] and NanI can induce the release of free sialic acids from enterocyte-like Caco-2 cells [33]. Since C. perfringens possesses a sialic acid utilization system, similar NanI-generated sialic acid in the intestines should be useful for in vivo growth of this bacterium.

Earlier studies demonstrated that NanI production also impacts the production of several C. perfringens toxins $[34,37,57]$. Specifically, inactivating the nanI gene in C. perfringens type A strain 13 caused a slight increase in supernatant activities of alpha toxin and perfringolysin O (PFO), which are contributors to gas gangrene [37], but their role (if any) in most intestinal infections, particularly human infections, is unsettled [3]. However, it was later demonstrated that inactivating the nanI gene in type D strain CN3718 reduced ETX toxin production in vitro and that either genetic or physical complementation could recover this ETX production [57]. NanI effects on ETX production involved reductions in both $\operatorname{cod} Y$ and $c c p A$ transcript levels, suggesting a model whereby NanI generates sialic acid release in the intestines and that free sialic acid then alerts a type D strain of its presence in the intestines, making it worthwhile for this bacterium to upregulate ETX production to induce disease.

There have been only limited studies to date on the effects of $C$. perfringens sialidase on toxin action, particularly on those toxins important for intestinal infections. An exception is ETX, where conclusions from early studies had been contradictory. One early study [58] reported that sialidases enhance ETX cytotoxicity towards Madin-Derby Canine Kidney (MDCK) epithelial cells, while a second study [59] reported that pretreating synaptosomal membranes with sialidases lowers their subsequent ETX binding levels. Recently a study used purified NanI, as well as nanI, nanJ, or nanH single null mutants, a nanI/nanJ double null mutant, and a triple sialidase null mutant, to study possible sialidase enhancement of ETX action [34]. Results obtained [34] conclusively showed that NanI sialidase increases the ETX sensitivity of MDCK cells and that this effect involves an increase in ETX binding levels. The mechanism of this enhancement is not yet clear but it could involve either NanI increasing the exposure of ETX receptors on the host cell surface or NanI modifying the host cell 
surface charge to increase binding of this toxin. Whether a similar effect extends to other $C$. perfringens toxins active during intestinal infections is not yet clear.

Only limited studies have addressed the mechanism of $C$. perfringens adherence to host cells and tissues [60-62]. For example, the adhesins used by C. perfringens in vivo remain unclear. However, some strains produce a collagen adhesion protein (CNA), and/or fibronectin binding proteins (FbpA, $\mathrm{FbpB})$ that have been implicated in adhesion and colonization [60-63].

Sialidases are known to contribute to the ability of some bacterial pathogens to adhere to host cells, tissues, or mucosal surfaces in the airways or intestines [30]. A recent in vitro study suggests sialidase contributions to C. perfringens intestinal adherence [34]. That study [34] demonstrated that type D strain CN3718 attaches to cultured Caco-2 enterocyte-like cells. This adhesion was specific since this intestinal disease strain exhibited significantly less adherence to fibroblasts and kidney cells [34]. Interestingly, the attachment of CN3718 to Caco-2 cells is greatly facilitated by NanI production (Figure 3). Wild-type CN3718 exhibited much more adherence to Caco-2 cells compared to an isogenic triple mutant strain that does not produce any sialidase. Complementation studies showed that, of the three sialidases made by $\mathrm{CN} 3718$, restoring NanI production to the triple sialidase mutant yielded the greatest enhancement of adherence. That result indicated that NanI is of prime importance for sialidase enhancement of CN3718 attachment to Caco-2 cells [34].

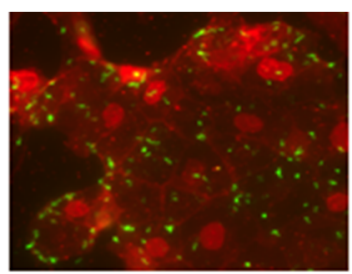

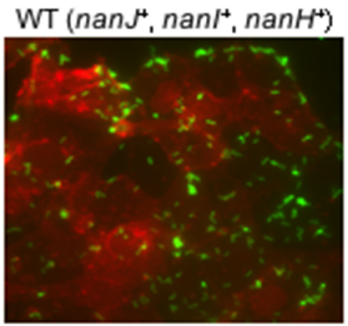

Complementing (nans, nanl+, nanH)

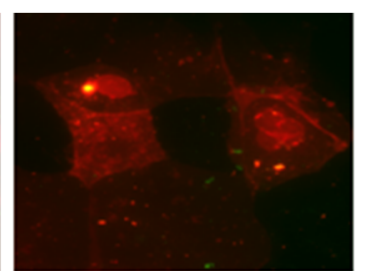

Mutant (nanf, nanl, nanH)

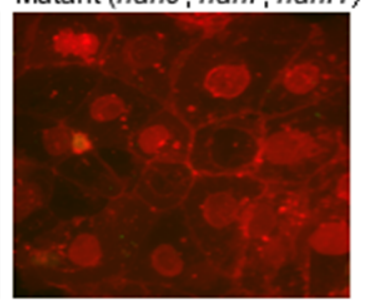

Negative Control

Figure 3. Adhesion of C. perfringens CN3718 to Caco-2 cells [34]. Caco-2 cells were incubated for $2 \mathrm{~h}$ at $37^{\circ} \mathrm{C}$ under anaerobic conditions. C. perfringens $\mathrm{CN} 3718$ produces all three sialidases. This wild-type (WT) strain, and a complementing strain that produces only NanI, attach very well to Caco-2 cells as detected by immunofluorescence microscopy $(600 \times)$. In comparison, only a few cells of an isogenic mutant with all three sialidase genes disrupted were able to attach to Caco- 2 cells. Furthermore, when the triple mutant was complemented to produce only NanJ or $\mathrm{NanH}$, those bacteria remained poorly adherent (not shown). Green: C. perfringens; Red: Caco-2 cells. Reproduced with permission from [34]. Creative Commons License 2011, Copyright J. Li and B.A. McClane.

However, NanI itself does not appear to be a significant adhesin since the CN3718 codY null mutant shows $50 \%$ less adherence to Caco-2 cells compared to wild-type CN3718, despite this mutant producing the same levels of NanI as the wild-type strain [45]. This result suggests a two-step adherence mechanism. First, secreted NanI modifies the Caco-2 enterocyte-cell surface, which then allows the unknown adhesin present on the surface of $C$. perfringens to more easily bind to the still unidentified receptor(s) on the enterocyte-cell surface.

Sialidases may enhance C. perfringens adherence to host cells via both nonspecific and specific mechanisms. Sialic acids, typically present at the distal ends of carbohydrate chains, are negatively charged carbohydrates $[28,30]$. In addition, terminal sialic acids promote endothelial barrier integrity, 
so treatment of epithelial monolayers with $C$. perfringens sialidases may lead to barrier disruption and may increase access for $C$. perfringens so they can adhere [64]. Therefore, nonspecific effects of secreted NanI on both charge and epithelial barrier integrity could help to increase toxin binding and C. perfringens colonization. A C. perfringens sialidase (not specified) has, in fact, been shown to affect barrier resistance in some, but not all, host cells [64]. However, for some cell lines, nonspecific effects do not appear to completely explain NanI enhancement of C. perfringens adherence. As mentioned, C. perfringens CN3718 exhibits much greater adhesion for certain mammalian cells, such as Caco-2 and HT-29 intestinal cell lines, than for cell lines of nonintestinal origin [34]. Similarly, the toxins involved in C. perfringens intestinal infections only bind to and affect certain cells. Those observations suggest that NanI can also have specific effects in promoting C. perfringens adherence and toxin binding. This could involve specific effects of NanI sialidase on modifying host cell surface adhesins/toxin receptors and/or trimming back nearby molecules to better unmask the adhesin or toxin receptor on host cell surfaces.

When C. perfringens causes diseases originating in the intestines, its secreted proteins come into contact with host proteases, such as trypsin, that are present in the intestinal lumen. While some C. perfringens proteins, e.g., CPB, are highly sensitive to intestinal proteases [16], other proteins secreted by this bacterium are proteolytically-activated in the intestines. A prime example of a proteolytically-activated C. perfringens protein is ETX, which is initially produced as an inactive prototoxin and then activated by intestinal trypsin, chymotrypsin, and carboxypeptidases that remove $\mathrm{N}$ - and C-terminus residues from the prototoxin [65]. Similarly, trypsin or chymotrypsin treatment of $\mathrm{CPE}$ also increases cytotoxicity, in this case by removing $\mathrm{N}$-terminal sequences from the native CPE protein to facilitate toxin oligomerization during pore formation [66]. Interestingly, NanI (but not NanJ or $\mathrm{NanH}$ ) can also be proteolytically-activated by trypsin [33,34]. The increased enzymatic activity of trypsin-activated NanI was shown to be substrate-specific [33]. Collectively, these observations suggest that trypsin activation of NanI may contribute to C. perfringens intestinal diseases.

While NanI can enhance C. perfringens adherence and toxin binding, typical type A FP strains, i.e., those with a chromosomal cpe gene, do not carry the nanI gene. Furthermore, those typical FP strains possess very low exosialidase activities [36]. The limited production of sialidases by type A FP strains obviously does not hinder their ability to cause food-borne intestinal diseases. Unlike the typical FP strains, nearly all type A AAD strains do carry a nanI gene. When a nanI null mutant of a type A AAD strain was characterized, this nanI null mutant was shown to enter the sporulation cycle earlier and to produce more CPE than its wild-type parent. This finding supports the dispensability of NanI for the typical FP strains, which cause an acute disease. During the acute FP, NanI would not be needed for nutritional purposes or to enhance bacteria adherence or colonization since the typical FP strains are ingested in very large numbers in contaminated food, then quickly sporulate in the intestines to produce CPE, induce diarrhea via that CPE, and exit from the intestines via the flushing action of diarrhea (Figure 4) [36]. In contrast, type A AAD or sporadic diarrhea (SD) strains with a plasmid cpe gene cause chronic gastrointestinal disease lasting up to several weeks, so in vivo growth and colonization is important for their persistence. Those Type A AAD or SD strains may use NanI for their growth and colonization, as necessary to achieve the persistence required to cause a chronic diarrhea (Figure 4) [36]. 
(A)

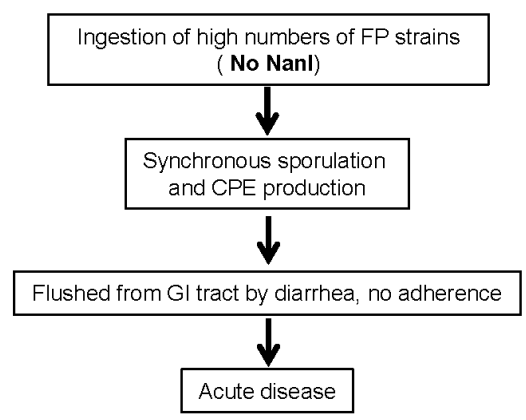

(B)

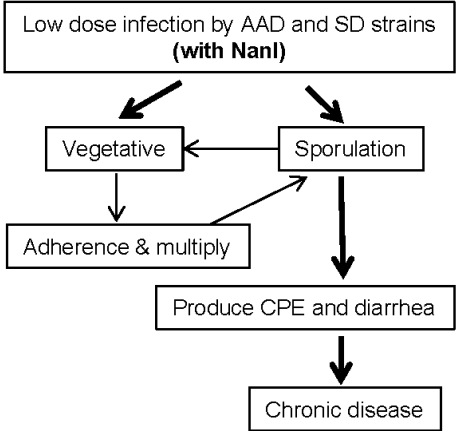

Figure 4. Possible models for acute C. perfringens type A FP vs. chronic gastrointestinal diseases like AAD. (A) C. perfringens type A FP does not require NanI production because strains are ingested in large amounts, sporulate in vivo to produce CPE and are then quickly removed from the intestines by diarrhea. (B) C. perfringens type A AAD does involve NanI production, which may promote adherence and colonization, as required for chronic diarrhea. Modified with permission from [36]. Copyright 2014 American Society for Microbiology.

\section{Sialidase Inhibitors: Potential Therapeutic Agents?}

As described above, sialidases may offer several contributions to C. perfringens pathogenesis, particularly during intestinal infections. C. perfringens diseases are challenging to treat with antibiotics because already-synthesized toxins will continue to work even after the administration of antibiotics. Similarly, it is often difficult to treat or prevent $C$. perfringens infections with vaccines or neutralizing antibodies because some individual strains produce multiple (up to five) different toxins $[2,3]$. Those factors suggest that sialidase inhibitors represent interesting potential candidates for drug development.

Sialidase inhibitors have proven to be efficacious for the treatment of some infectious disease [67]. The most notable clinical application of sialidase inhibitors are several sialidase inhibitors (Zanamivir, Oseltamivir, Peramivir (Rapivab)) that are currently used for treating infections involving influenza virus [67]. However, there is also precedent for sialidase inhibitors interfering with bacterial growth and adhesion. Oseltamivir reduced the growth and adherence of Tannerella forsythia, which causes periodontitis, by two- to three-fold [68].

Two classic sialidase inhibitors, i.e., Siastatin B (SB) and N-acetyl-2,3-dehydro-2-deoxyneuraminic acid (NADNA), have been tested against $C$. perfringens $[33,36,57,69,70]$. SB, a broad-spectrum sialidase inhibitor isolated from a Streptomyces spp. culture, is an unusual 6-acetamido-3-piperidinecarboxy late [70]. NADNA is an analogue of 2-deoxy-2,3-didehydro-N-acetylneuraminic acid that is modified at the C-4 position. NADNA can inhibit the sialidase activity from influenza viruses A and B, parainfluenza 2 virus, Vibrio cholerae, Arthrobacter ureafaciens, C. perfringens, and sheep liver [69].

A recent study measured the effects of SB and NADNA on sialidase activities of overnight culture supernatants of $C$. perfringens CN3718 mutants ENanJ, ENanI, or ENanH, which contain natural levels of only NanJ, NanI, or NanH, respectively [33]. NanI was the most sensitive sialidase to both NADNA and $\mathrm{SB}$, while NanH was the least sensitive sialidase to these inhibitors; $\mathrm{IC}_{50} \mathrm{~s}$ for these inhibitors are shown in Table 3 [33]. 
Table 3. Effect of sialidase inhibition on sialidase activity $\left(\mathrm{IC}_{50}\right)$.

\begin{tabular}{ccc}
\hline Sample & NADNA $\left(\mathbf{I C}_{\mathbf{5 0}}\right)$ & Siastatin $\mathbf{B}\left(\mathbf{I C}_{\mathbf{5 0}}\right)$ \\
\hline CN3718 & $18.9 \mu \mathrm{M}$ & $42.2 \mu \mathrm{M}$ \\
ENanJ & $12.4 \mu \mathrm{M}$ & $15.1 \mu \mathrm{M}$ \\
ENanI & $13.4 \mu \mathrm{M}$ & $27.5 \mu \mathrm{M}$ \\
ENanH & $44.6 \mu \mathrm{M}$ & $50.9 \mu \mathrm{M}$
\end{tabular}

Reproduced with permission from [33]. Copyright 2014 American Society for Microbiology.

It was also shown that the adherence of $C$. perfringens AAD strain F4969 to Caco-2 cells can be reduced by either SB or NADNA [36]. In addition, both NADNA and SB efficiently inhibited sialidase activity in bacterial cell-free supernatants collected from C. perfringens type D strain CN3718. Consistent with those results, CN3718 cultures grown in the presence of the SB inhibitor also exhibited substantially reduced culture sialidase activity and ETX production. However, the NADNA sialidase inhibitor did not inhibit sialidase activity in CN3718 cultures, even at very high doses of the inhibitor. In the presence of NADNA, CN3718 cultures still possessed strong sialidase activity and also made the same amounts of ETX as wild-type CN3718 grown in the absence of any sialidase inhibitor [57]. Collectively, these results suggest that at least some sialidase inhibitors may be potentially useful therapeutics against $C$. perfringens infections.

\section{Concluding Remarks and Future Directions}

Most studies of C. perfringens virulence have, correctly, focused on toxin contributions. Only a few in vivo studies have thus far examined the role of exoenzymes in C. perfringens pathogenesis and their results have been equivocal. However, those studies examined the possible role of sialidases in gas gangrene. Recent in vitro studies suggest that sialidases, particularly NanI, may be important contributors to $C$. perfringens intestinal infections. Those contributions could include upregulated production of some toxins relevant to intestinal infections, enhanced binding and activity of some of those toxins, increased adherence of $C$. perfringens to host cells, and possibly generation of substrates for growth and metabolism [33,34,36,57]. There is a clear need to move these promising findings into appropriate animal models to firmly evaluate sialidase contributions to $C$. perfringens virulence.

In vitro studies also suggest that some sialidase inhibitors might be useful therapeutics for treating C. perfringens infections originating in the intestines [57]. This possibility also needs to be evaluated in animals. Identification of more potent inhibitors of $C$. perfringens sialidases may be helpful for those studies.

Acknowledgments: Preparation of this review was supported, in part, by a grant from the National Institute of Allergy and Infectious Disease (R21 AI125796-1).

Conflicts of Interest: The authors declare no conflict of interest.

\section{References}

1. McClane, B.A.; Robertson, S.L.; Li, J. Clostridium perfringens. In Food Microbiology: Fundamentals and Frontiers, 4th ed.; Doyle, M.P., Buchanan, R.L., Eds.; ASM Press: Washington, DC, USA, 2013; pp. 465-489.

2. McClane, B.A.; Uzal, F.A.; Miyakawa, M.F.; Lyerly, D.; Wilkins, T.D. The enterotoxic clostridia. In The Prokaryotes, 3rd ed.; Dworkin, M., Falkow, S., Rosenburg, E., Schleifer, H., Stackebrandt, E., Eds.; Springer: New York, NY, USA, 2006; pp. 688-752.

3. Uzal, F.A.; Freedman, J.C.; Shrestha, A.; Theoret, J.R.; Garcia, J.; Awad, M.M.; Adams, V.; Moore, R.J.; Rood, J.I.; McClane, B.A. Towards an understanding of the role of Clostridium perfringens toxins in human and animal disease. Future Microbiol. 2014, 9, 361-377. [CrossRef] [PubMed]

4. Rood, J.I. Clostridium perfringens and histotoxic disease. In The prokaryotes: A Handbook on the Biology of Bacteria, 3rd ed.; Dworkin, M., Falkow, S., Rosenberg, E., Schleifer, K.-H., Stackebrandt, E., Eds.; Springer: New York, NY, USA, 2007; Volume 4, pp. 753-770. 
5. Titball, R.W.; Rood, J.I. Clostridium pefringens: Wound indections. In Molecular Medical Microbiology; Sussman, M., Ed.; Academic Press: London, UK, 2002; pp. 1875-1903.

6. Uzal, F.A.; Vidal, J.E.; McClane, B.A.; Gurjar, A.A. Clostridium perfringens toxins involved in mammalian veterinary diseases. Open toxinol. J. 2010, 2, 24-42. [CrossRef]

7. Songer, J.G. Clostridial enteric diseases of domestic animals. Clin. Microbiol. Rev. 1996, 9, 216-234. [PubMed]

8. Hatheway, C. Toxigenic clostridia. Clin. Microb. Rev. 1990, 3, 66-76. [CrossRef]

9. McDonel, J.L. Toxins of Clostridium perfringens types A, B, C, D, and E. In Pharmacology of Bacterial Toxins; Dorner, F., Drews, H., Eds.; Pergamon Press: Oxford, UK, 1986; pp. 477-517.

10. Petit, L.; Gilbert, M.; Popoff, M. Clostridium perfringens: Toxinotype and genotype. Trends Microbiol. 1999, 7 , 104-110. [CrossRef]

11. Animoto, K.; Noro, T.; Oishi, E.; Shimizu, M. A novel toxin homologous to large clostridial cytotoxins found in culture supernatant of Clostridium perfringens type C. Microbiology 2007, 153, 1198-1206. [CrossRef] [PubMed]

12. Keyburn, A.L.; Boyce, J.D.; Vaz, P.; Bannam, T.L.; Ford, M.E.; Parker, D.; Di Rubbo, A.; Rood, J.I.; Moore, R.J. NetB, a new toxin that is associated with avian necrotic enteritis caused by Clostridium perfringens. PLoS Pathog. 2008, 4, e26. [CrossRef] [PubMed]

13. Mehdizadeh Gohari, I.; Parreira, V.R.; Timoney, J.F.; Fallon, L.; Slovis, N.; Prescott, J.F. NetF-positive Clostridium perfringens in neonatal foal necrotising enteritis in kentucky. Vet. Rec. 2016, 178, 216. [CrossRef] [PubMed]

14. Yonogi, S.; Matsuda, S.; Kawai, T.; Yoda, T.; Harada, T.; Kumeda, Y.; Gotoh, K.; Hiyoshi, H.; Nakamura, S.; Kodama, T.; et al. BEC, a novel enterotoxin of Clostridium perfringens found in human clinical isolates from acute gastroenteritis outbreaks. Infect Immun. 2014, 82, 2390-2399. [CrossRef] [PubMed]

15. Irikura, D.; Monma, C.; Suzuki, Y.; Nakama, A.; Kai, A.; Fukui-Miyazaki, A.; Horiguchi, Y.; Yoshinari, T.; Sugita-Konishi, Y.; Kamata, Y. Identification and characterization of a new enterotoxin produced by Clostridium perfringens isolated from food poisoning outbreaks. PLoS ONE 2015, 10, e0138183. [CrossRef] [PubMed]

16. Sayeed, S.; Uzal, F.A.; Fisher, D.J.; Saputo, J.; Vidal, J.E.; Chen, Y.; Gupta, P.; Rood, J.I.; McClane, B.A. Beta toxin is essential for the intestinal virulence of Clostridium perfringens type $\mathrm{C}$ disease isolate $\mathrm{CN} 3685$ in a rabbit ileal loop model. Mol. Microbiol. 2008, 67, 15-30. [CrossRef] [PubMed]

17. Garcia, J.P.; Adams, V.; Beingesser, J.; Hughes, M.L.; Poon, R.; Lyras, D.; Hill, A.; McClane, B.A.; Rood, J.I.; Uzal, F.A. Epsilon toxin is essential for the virulence of Clostridium perfringens type D infection in sheep, goats and mice. Infect Immun. 2013, 81, 2405-2414. [CrossRef] [PubMed]

18. Ma, M.; Gurjar, A.; Theoret, J.R.; Garcia, J.P.; Beingesser, J.; Freedman, J.C.; Fisher, D.J.; McClane, B.A.; Uzal, F.A. Synergistic effects of Clostridium perfringens enterotoxin and beta toxin in rabbit small intestinal loops. Infect Immun. 2014, 82, 2958-2970. [CrossRef] [PubMed]

19. Ma, M.; Li, J.; McClane, B.A. Genotypic and phenotypic characterization of Clostridium perfringens isolates from darmbrand cases in post-World War II Germany. Infect Immun. 2012, 80, 4354-4363. [CrossRef] [PubMed]

20. Lawrence, G.W. The pathogenesis of enteritis necroticans. In The Clostridia: Molecular Genetics and Pathogenesis; Rood, J.I., McClane, B.A., Songer, J.G., Titball, R.W., Eds.; Academic Press: London, UK, 1997; pp. $198-207$.

21. Johnson, S.; Gerding, D.N. Enterotoxemic infections. In The Clostridia: Molecular Biology and Pathogenesis; Rood, J.I., McClane, B.A., Songer, J.G., Titball, R.W., Eds.; Academic Press: London, UK, 1997; pp. 117-140.

22. Freedman, J.C.; Shrestha, A.; McClane, B.A. Clostridium perfringens enterotoxin: Action, genetics, and translational applications. Toxins 2016, 8. [CrossRef] [PubMed]

23. Centers for Disease Control and Prevention (CDC). Posting date. CDC estimates of foodborne illness in the United States: Clostridium perfringens. Available online: http://www.cdc.gov/foodsafety/diseases/ clostridium-perfringens.html (accessed on 28 July 2016).

24. Caserta, J.A.; Robertson, S.L.; Saputo, J.; Shrestha, A.; McClane, B.A.; Uzal, F.A. Development and application of a mouse intestinal loop model to study the in vivo action of Clostridium perfringens enterotoxin. Infect. Immun. 2011, 79, 3020-3027. [CrossRef] [PubMed]

25. Carman, R.J. Clostridium perfringens in spontaneous and antibiotic-associated diarrhoea of man and other animals. Rev. Med. Microbiol. 1997, 8 (Suppl. S1), S43-S45. [CrossRef] 
26. Li, J.; Paredes-Sabja, D.; Sarker, M.; Mcclane, B. Clostridium perfringens sporulation and sporulation-associated toxin production. Microbiol. Spectr. 2016, 4. [CrossRef]

27. Li, J.; McClane, B.A. A novel small acid soluble protein variant is important for spore resistance of most Clostridium perfringens food poisoning isolates. PLoS Pathog. 2008, 4, e1000056. [CrossRef] [PubMed]

28. Severi, E.; Hood, D.W.; Thomas, G.H. Sialic acid utilization by bacterial pathogens. Microbiology 2007, 153, 2817-2822. [CrossRef] [PubMed]

29. Traving, C.; Schauer, R. Structure, function and metabolism of sialic acids. Cell. Mol. Life Sci. 1998, 54, 1330-1349. [CrossRef] [PubMed]

30. Lewis, A.L.; Lewis, W.G. Host sialoglycans and bacterial sialidases: A mucosal perspective. Cell. Microbiol. 2012, 14, 1174-1182. [CrossRef] [PubMed]

31. Vimr, E.R.; Kalivada, K.A.; Deszo, E.L.; Steenbergen, S.M. Diversity of microbial sialic acid metabolism. Microbiol. Molec Biol. Rev. 2004, 68, 132-153. [CrossRef]

32. Rohmer, L.; Hocquet, D.; Miller, S.I. Are pathogenic bacteria just looking for food? Metabolism and microbial pathogenesis. Trends Microb. 2011, 19, 341-348. [CrossRef] [PubMed]

33. Li, J.; McClane, B.A. The sialidases of Clostridium perfringens type D strain CN3718 differ in their properties and sensitivities to inhibitors. Appl. Environ. Microbiol. 2014, 80, 1701-1709. [CrossRef] [PubMed]

34. Li, J.; Sayeed, S.; Robertson, S.; Chen, J.; McClane, B.A. Sialidases affect the host cell adherence and epsilon toxin-induced cytotoxicity of Clostridium perfringens type D strain CN3718. PLoS Pathog. 2011, 7, e1002429. [CrossRef] [PubMed]

35. Boraston, A.B.; Ficko-Blean, E.; Healey, M. Carbohydrate recognition by a large sialidase toxin from Clostridium perfringens. Biochemistry 2007, 46, 11352-11360. [CrossRef] [PubMed]

36. Li, J.; McClane, B.A. Contributions of NanI sialidase to Caco-2 cell adherence by Clostridium perfringens type $A$ and $C$ strains causing human intestinal disease. Infect Immun. 2014, 82, 4620-4630. [CrossRef] [PubMed]

37. Chiarezza, M.; Lyras, D.; Pidot, S.J.; Flore-Diaz, M.; Awad, M.M.; Kennedy, C.L.; Cordner, L.M.; Phumoonna, T.; Poon, R.; Hughes, M.L.; et al. The NanI and NanJ sialidases of Clostridium perfringens are not essential for virulence. Infect Immun. 2009, 77, 4421-4428. [CrossRef] [PubMed]

38. Myers, G.S.; Rasko, D.A.; Cheung, J.K.; Ravel, J.; Seshadri, R.; DeBoy, R.T.; Ren, Q.; Varga, J.; Awad, M.M.; Brinkac, L.M.; et al. Skewed genomic variability in strains of the toxigenic bacterial pathogen, Clostridium perfringens. Genome Res. 2006, 16, 1031-1040. [CrossRef] [PubMed]

39. Shimizu, T.; Ohtani, K.; Hirakawa, H.; Ohshima, K.; Yamashita, A.; Shiba, T.; Ogasawara, N.; Hattori, M.; Kuhara, S.; Hayashi, H. Complete genome sequence of Clostridium perfringens, an anaerobic flesh-eater. Proc. Natl. Acad. Sci. USA 2002, 99, 996-1001. [CrossRef] [PubMed]

40. Therit, B.; Cheung, J.K.; Rood, J.I.; Melville, S.B. NanR, a transcriptional regulator that binds to the promoters of genes involved in sialic acid metabolism in the anaerobic pathogen Clostridium perfringens. PLoS ONE 2015, 10, e0133217. [CrossRef] [PubMed]

41. Ohtani, K.; Hirakawa, H.; Tashiro, K.; Yoshizawa, S.; Kuhara, S.; Shimizu, T. Identification of a two-component VirR/VirS regulon in Clostridium perfringens. Anaerobe 2010, 16, 258-264. [CrossRef] [PubMed]

42. Ohtani, K.; Shimizu, T. Regulation of toxin gene expression in Clostridium perfringens. Res. Microbiol. 2014, 166, 280-289. [CrossRef] [PubMed]

43. Hiscox, T.J.; Harrison, P.F.; Chakravorty, A.; Choo, J.M.; Ohtani, K.; Shimizu, T.; Cheung, J.K.; Rood, J.I. Regulation of sialidase production in Clostridium perfringens by the orphan sensor histidine kinase ReeS. PLOS ONE 2013, 8, e73525. [CrossRef] [PubMed]

44. Hiscox, T.J.; Chakravorty, A.; Choo, J.M.; Ohtani, K.; Shimizu, T.; Cheung, J.K.; Rood, J.I. Regulation of virulence by the RevR response regulator in Clostridium perfringens. Infect. Immun. 2011, 79, 2145-2153. [CrossRef] [PubMed]

45. Li, J.; Ma, M.; Sarker, M.R.; McClane, B.A. CodY is a global regulator of virulence-associated properties for Clostridium perfringens type D strain CN3718. mBio 2013, 4, e00770-e00713. [CrossRef] [PubMed]

46. Walters, D.M.; Stirewalt, V.L.; Melville, S.B. Cloning, sequence, and transcriptional regulation of the operon encoding a putative $\mathrm{N}$-acetyl-mannosamine-6-phosphate epimerase (nanE) and sialic acid lyase (nanA) in Clostridium perfringens. J. Bacteriol. 1999, 181, 4526-4532. [PubMed]

47. Olson, M.E.; King, J.M.; Yahr, T.L.; Horswill, A.R. Sialic acid catabolism in Staphylococcus aureus. J. Bacteriol. 2013, 195, 1779-1788. [CrossRef] [PubMed] 
48. Almagro-Moreno, S.; Boyd, E.F. Bacterial catabolism of nonulosonic (sialic) acid and fitness in the gut. Gut Microbes 2010, 1, 45-50. [CrossRef] [PubMed]

49. Almagro-Moreno, S.; Boyd, E.F. Sialic acid catabolism confers a competitive advantage to pathogenic Vibrio cholerae in the mouse intestine. Infect. Immun. 2009, 77, 3807-3816. [CrossRef] [PubMed]

50. Ng, K.M.; Ferreyra, J.A.; Higginbottom, S.K.; Lynch, J.B.; Kashyap, P.C.; Gopinath, S.; Naidu, N.; Choudhury, B.; Weimer, B.C.; Monack, D.M.; et al. Microbiota-liberated host sugars facilitate post-antibiotic expansion of enteric pathogens. Nature 2013, 502, 96-99. [CrossRef] [PubMed]

51. Ley, R.E. Harnessing microbiota to kill a pathogen: The sweet tooth of Clostridium difficile. Nat. Med. 2014, 20, 248-249. [CrossRef] [PubMed]

52. Brittan, J.; Bucheridge, T.; Finn, A.; Kadioglu, A.; Jenkinson, H. Pneumococcal neuraminidase A: An essential upper airway colonization factor for Streptococcus pneumoniae. Mol. Oral Microb. 2012, 27, 270-283. [CrossRef] [PubMed]

53. Awad, M.M.; Singleton, J.; Lyras, D. The sialidase NanS enhances non-TcsL mediated cytotoxicity of Clostridium sordellii. Toxins 2016, 8. [CrossRef] [PubMed]

54. Chang, D.E.; Smalley, D.J.; Tucker, D.L.; Leatham, M.P.; Norris, W.E.; Stevenson, S.J.; Anderson, A.B.; Grissom, J.E.; Laux, D.C.; Cohen, P.S.; et al. Carbon nutrition of Escherichia coli in the mouse intestine. Proc. Natl. Acad. Sci. USA 2004, 101, 7427-7432. [CrossRef] [PubMed]

55. Galen, J.E.; Ketley, J.M.; Fasano, A.; Richardson, S.H.; Wasserman, S.S.; Kaper, J.B. Role of Vibrio cholerae neuraminidase in the fuction of cholera toxin. Infect. Immun. 1992, 60, 406-415. [PubMed]

56. Jeong, H.G.; Oh, M.H.; Kim, B.S.; Lee, M.Y.; Han, H.J.; Choi, S.H. The capability of catabolic utilization of $\mathrm{N}$-acetylneuraminic acid, a sialic acid, is essential for Vibrio vulnificus pathogenesis. Infect. Immun. 2009, 77, 3209-3217. [CrossRef] [PubMed]

57. Li, J.; Freedman, J.C.; McClane, B.A. NanI sialidase, CcpA, and CodY work together to regulate epsilon toxin production by Clostridium perfringens type D strain CN3718. J. Bacteriol. 2015, 197, 3339-3353. [CrossRef] [PubMed]

58. Shimanoto, S.; Tamai, E.; Matsushita, O.; Minami, J.; Okabe, A.; Miyata, S. Changes in ganglioside content affect the binding of Clostridium perfringens epsilon-toxin to detergent-resistant membranes of Madin-Darby canine kidney cells. Microbiol. Immun. 2005, 49, 245-253. [CrossRef]

59. Nagahama, M.; Sakurai, J. High-affinity binding of Clostridium perfingens epsilon-toxin to rat brain. Infect. Immun. 1992, 60, 1237-1240. [PubMed]

60. Jost, B.H.; Billington, S.J.; Trinh, H.T.; Songer, J.G. Association of genes encoding beta2 toxin and a collagen binding protein in Clostridium perfringens isolates of porcine origin. Vet. Microbiol. 2006, 115, 173-182. [CrossRef] [PubMed]

61. Hitsumoto, Y.; Morita, N.; Yamazoe, R.; Tagomori, M.; Yamasaki, T.; Katayama, S. Adhesive properties of Clostridium perfringens to extracellular matrix proteins collagens and fibronectin. Anaerobe 2014, 25, 67-71. [CrossRef] [PubMed]

62. Katayama, S.; Tagomori, M.; Morita, N.; Yamasaki, T.; Nariya, H.; Okada, M.; Watanabe, M.; Hitsumoto, Y. Determination of the Clostridium perfringens-binding site on fibronectin. Anaerobe 2015, 34, 174-181. [CrossRef] [PubMed]

63. Wade, B.; Keyburn, A.L.; Haring, V.; Ford, M.; Rood, J.I.; Moore, R.J. The adherent abilities of Clostridium perfringens strains are critical for the pathogenesis of avian necrotic enteritis. Vet. Microbiol. 2016, 197, 53-61. [CrossRef]

64. Cioffi, D.L.; Pandey, S.; Alvarez, D.F.; Cioffi, E.A. Terminal sialic acids are an important determinant of pulmonary endothelial barrier integrity. Am. J. Physiol. Lung Cell. Mol. Physiol. 2012, 302, L1067-1077. [CrossRef] [PubMed]

65. Freedman, J.C.; Li, J.; Uzal, F.A.; McClane, B.A. Proteolytic processing and activation of Clostridium perfringens epsilon toxin by caprine small intestinal contents. mBio 2014, 5, e01994-e01914. [CrossRef] [PubMed]

66. Hanna, P.C.; Wieckowski, E.U.; Mietzner, T.A.; McClane, B.A. Mapping functional regions of Clostridium perfringens type A enterotoxin. Infect. Immun. 1992, 60, 2110-2114. [PubMed]

67. Streicher, H. Inhibition of microbial sialidases-what has happened beyond the influenza virus? Curr. Med. Chem. Anti-Infect. Agents 2004, 3, 149-161. [CrossRef]

68. Roy, S.; Honma, K.; Douglas, C.W.; Sharma, A.; Stafford, G.P. Role of sialidase in glycoprotein utilization by Tannerella forsythia. Microbiology 2011, 157, 3195-3202. [CrossRef] [PubMed] 
69. Holzer, C.T.; von Itzstein, M.; Jin, B.; Pegg, M.S.; Stewart, W.P.; Wu, W.Y. Inhibition of sialidases from viral, bacterial and mammalian sources by analogues of 2-deoxy-2,3-didehydro- $N$-acetylneuraminic acid modified at the C-4 position. Glycoconj. J. 1993, 10, 40-44. [CrossRef] [PubMed]

70. Knapp, S.; Zhao, D. Synthesis of the sialidase inhibitor siastatin B. Org. Lett. 2000, 2, 4037-4040. [CrossRef] [PubMed]

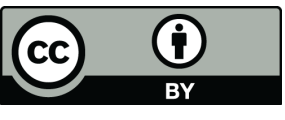

(C) 2016 by the authors; licensee MDPI, Basel, Switzerland. This article is an open access article distributed under the terms and conditions of the Creative Commons Attribution (CC-BY) license (http://creativecommons.org/licenses/by/4.0/). 